Haya: The Saudi Journal of Life Sciences

Abbreviated Key Title: Haya Saudi J Life Sci

ISSN 2415-623X (Print) |ISSN 2415-6221 (Online)

Scholars Middle East Publishers, Dubai, United Arab Emirates

Journal homepage: https://saudijournals.com

Review Article

\title{
Melatonin as an Anti-Cancer Molecule: A Retrospective Perspective
}

\author{
Somenath Ghosh
}

Assistant Professor, Department of Zoology, Rajendra PG College, Jai Prakash University, Chapra-841301, India

DOI: $10.36348 /$ sjls.2020.v05i12.005 $\quad$ | Received: 26.09.2020 | Accepted: 06.10.2020 | Published: 14.12 .2020

*Corresponding author: Dr. Somenath Ghosh

Abstract

Etymologically, melatonin (N-acetyl-5-methoxytryptamine) can be traced back to the origin of life. The first origin of melatonin was detected in cyano-bacteria. As considering the endosymbiont hypothesis, cyanobacteria were engulfed by the animal and plant cells. Later on, these bacteria performed the roles of mitochondria and chloroplastids in animals and plant cells respectively. Inner matrices of these organelles are having melatonin and probable function of this intraorganelle melatonin is to protect the organelles from the detrimental effects from free radicals (Reactive Oxygen Species; ROSs and Reactive Nitrogen Species; RNSs). In higher animals, melatonin is synthesized and secreted by the pineal gland mainly during the night, since light exposure suppresses its production. Other than pineal gland, melatonin is secreted from several different organs like retina, gastro-intestinal tract. The secretion of this hormone is regulated by several environmental factors like photo-period, temperature, humidity etc. Melatonin can exert its function either by as a free molecule or by its membrane bound receptors MT1 and MT2 respectively. Modulations of melatonin receptors results in stimulation of apoptosis, regulation of pro-survival signaling, inhibition on angiogenesis, metastasis, and induction of epigenetic alteration. Melatonin could also be utilized as adjuvant of cancer therapies, through reinforcing the therapeutic effects and reducing the side effects of chemotherapies or radiation. Melatonin could be an excellent candidate for prevention and treatment of several cancers, such as breast cancer, prostate cancer, gastric cancer and colorectal cancer. This review summarized the anticancer efficacy of melatonin, based on the results of epidemiological, experimental and clinical studies.

Keywords: Anti-cancer, Anti-oxidant, Animal, Breast-cancer, Cancer, Colorectal-cancer, Etymology, Gastric-cancer, Hormone, Melatonin, MT1, MT2, Plant, Prostate-cancer, Receptor, ROS, RNS.

Copyright (C) 2020 The Author(s): This is an open-access article distributed under the terms of the Creative Commons Attribution 4.0 International License (CC BY-NC 4.0) which permits unrestricted use, distribution, and reproduction in any medium for non-commercial use provided the original author and source are credited.

\section{INTRODUCTION}

\section{Melatonin synthesis and pathophysiology}

Melatonin $\quad(N$-acetyl-5-methoxytryptamine $)$ was first isolated and identified by [1]. It is the major neurohormone secreted during the dark hours at night by the vertebrate pineal gland. Tryptophan serves as the precursor for melatonin biosynthesis, and is taken up from the circulation and then converted into serotonin. Serotonin is then converted into $N$-acetylserotonin by the enzyme arylalkylamine- $N$-acetyl transferase (AANAT) while $N$-acetylserotonin is metabolized into melatonin by the enzyme hydroxyindole- $O$ methyltransferase (HIOMT, 2) Once formed, melatonin is released into the capillaries and in higher concentrations into the cerebrospinal fluid [3] and is then rapidly distributed to most body tissues [4]. Intravenously administered melatonin exhibits biexponential decay with a first distribution half-life of $2 \mathrm{~min}$ and a second metabolic half-life of $20 \mathrm{~min}$ [5]. Circulating melatonin is metabolized mainly in the liver where it is first hydroxylated by cytochrome $\mathrm{P}_{450}$ monooxygenases and then conjugated with sulfate to form 6sulfatoxymelatonin [6]. Melatonin is also metabolized by oxidative pyrole-ring cleavage into kynuramine derivatives. The primary cleavage product is $N^{1}$-acetyl$N^{2}$-formyl-5-methoxykynuramine (AFMK), which is deformylated, either by arylamine formamidase or hemoperoxidases to $N^{1}$-acetyl-5-methoxykynuramine $[7,8]$.

Some evidence has suggested that pyrrole ring cleavage contributes to about one-third of the total melatonin catabolism. It has been proposed that AFMK is the primitive and primary active metabolite of melatonin [9]. The circadian pattern of pineal melatonin secretion is regulated by the biological clock that resides in mammals within the hypothalamic suprachiasmatic nucleus $(\mathrm{SCN})$ of the hypothalamus. Lesions in the SCN abolish the rhythm of pineal melatonin production in mammals [10]. The $\mathrm{SCN}$ is synchronized to the environmental light-dark cycle by 
light perceived by the retina, acting mainly on a subgroup of retinal ganglion cells (RGCs) that contain the photo-pigment melanopsin [11]. These RGCs connect to the SCN via the retino-hypothalamic tract. The SCN regulates pineal gland's function through a polysynaptic network involving the paraventricular nucleus of the hypothalamus. Descending polysynaptic fibers from these regions project through the medial forebrain bundle and reticular formation projects towards the inter-medio-lateral horns of the cervical segments of the spinal cord [12]. Postganglionic sympathetic fibers from the superior cervical ganglia reach the pineal gland and regulate melatonin biosynthesis through the presynaptic release of norepinephrine (NE). NE release occurs during the "night" portion of the circadian pacemaker cycle provided that this occurs in a dark environment.

Activation of the pineal $\beta$-adrenergic receptors by $\mathrm{NE}$ results in increased 3', 5'-cyclic adenosine monophosphate (cAMP) concentration that promotes the biosynthesis of melatonin [13]. $\alpha 1$-Adrenergic receptors potentiate $\beta$-adrenergic activity by producing a sharp increase in intracellular $\mathrm{Ca}^{2+}$ and activation of protein kinase $\mathrm{C}(\mathrm{PKC})$ and of prostaglandin synthesis [14-16]. The subcellular mechanisms involved in increase and turnoff of AANAT activity have been elucidated in great detail [17]. Cyclic AMP stimulates AANAT expression and phosphorylation via protein kinase A, which also allows AANAT to be stabilized by binding of 14-3-3 proteins [18, 19]. The nocturnal exposure to bright light suppresses melatonin production immediately by degradation of pineal AANAT [20]. It has now been demonstrated that melatonin is produced by many organs other than the pineal gland. These include the retina [21], gastrointestinal tract [22], skin [23], lymphocytes [24] and bone marrow [25]. Because pineal melatonin production occurs during the dark phase and is acutely suppressed by light, and, further, because melatonin is quickly cleared from the circulation following the cessation of its production, the time and duration of the melatonin peak reflect the environmental night period [26]. Plasma melatonin exhibits a circadian rhythm with high levels at night, and low levels during the day, attaining peak concentrations of plasma melatonin between 02:00 and 04:00 h. Longer nights are associated with a longer duration of melatonin secretion [26]. Hence melatonin is a signal of darkness that encodes time-of-day and length-of - day information to the brain including the $\mathrm{SCN}$, brain and peripheral organs [27]. In mammals, melatonin is critical for the regulation of seasonal changes for various physiological, neuroendocrine and reproductive functions [28]. These actions of melatonin are processed in nuclei of the hypothalamus and in the pars tuberalis (PT) of the pituitary [29]. The following illustration as suggested by Gupta et al., [30] shows the synthesis and regulation of synthesis of melatonin.

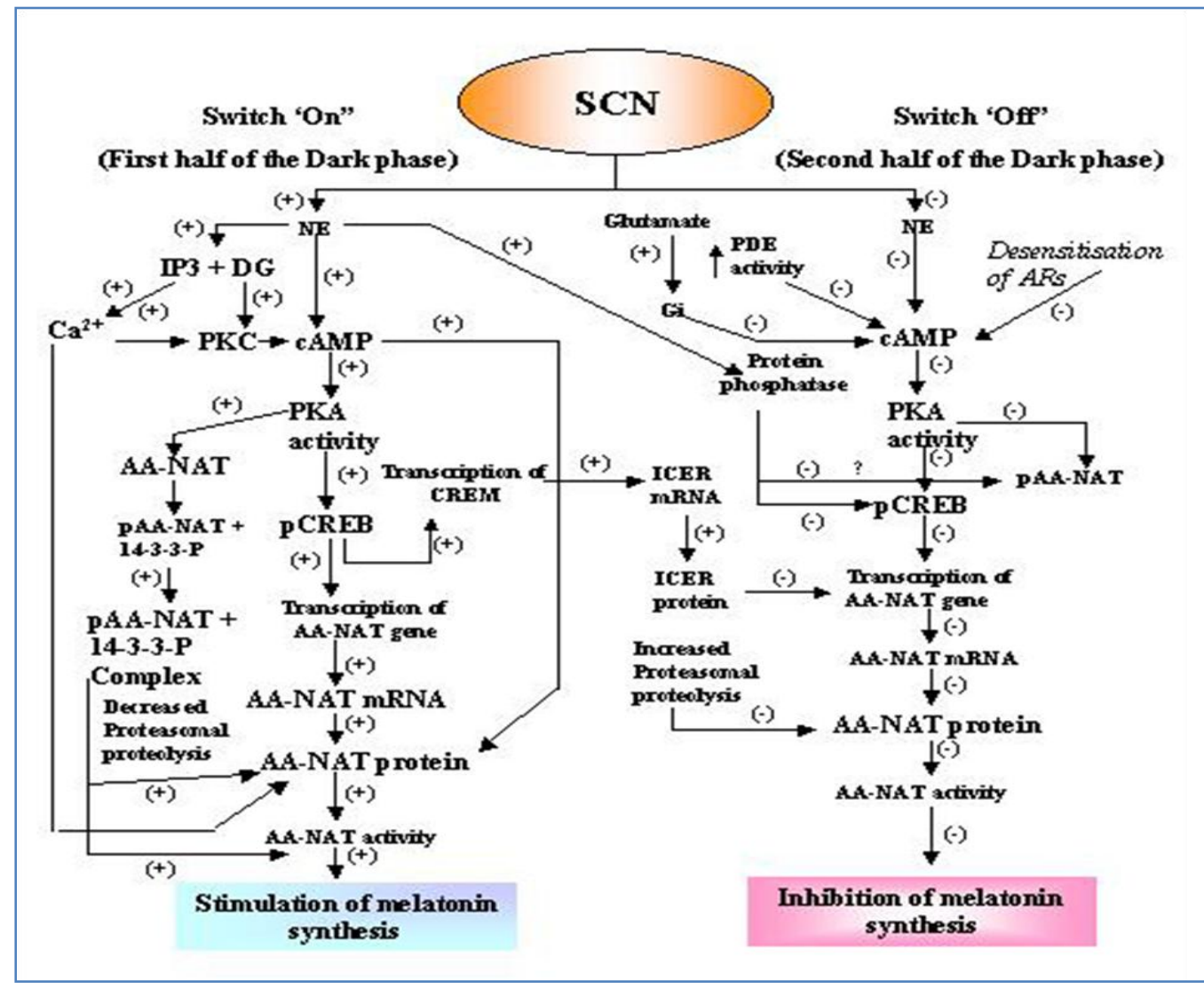

Fig-1: Synthesis and regulation of synthesis of melatonin (Gupta et al., 1995) 


\section{Different functions of melatonin}

Melatonin is reported to have a role in sleep initiation as the trigger for opening the circadian "sleep gate", acting as a sleep regulator [27]. Other actions of the hormone include inhibition of dopamine (DA) release in the hypothalamus and retina [31], involvement in the aging process [32] and pubertal development [33], blood pressure control [34, 35], freeradical scavenging [36] and regulation of the immune response [24]. If given during the day, when it is not present endogenously, melatonin has soporific effects which resemble its action at night, i.e., it lowers body temperature and induces fatigue while concomitantly producing a brain activation pattern resembling that which occurs during sleep [37].

Melatonin production decreases with age and in certain diseases, e.g., certain malignancies, Alzheimer's disease (AD) and cardiovascular disease $[38,39]$. This decrease in melatonin output has been linked to insomnia in older patients [40] and to a higher prevalence of cancer [41].

\section{MELATONIN AND CANCER \\ Overview of role of melatonin in cancer development}

Melatonin has been known to affect the growth of tumors, and its secretion corresponds with the presence of light and a circadian rhythm. The relationship between melatonin secretion, light, and cancer suggests that an increase in the prevalence of various types of cancer corresponds with industrialization and exposure to more forms of artificial light, thus resulting in lower levels of nocturnal melatonin. In animals, pineal suppression and pinealectomy stimulate the growth and metastasis of experimental cancers of the lung, liver, ovary, pituitary, and prostate. [42] Mouse studies using different melatonin administration protocols showed that the drug decreased the incidence and size of mammary adenocarcinomas and lung metastases [43].

\section{Melatonin and breast cancer}

Clinical evidence and research have also shown the prospective use of melatonin as a treatment for breast cancer patients. It was found that in women with breast cancer, the melatonin levels both in morning and evening were abnormal. Nocturnal melatonin levels were low while morning urine samples of breast cancer patients displayed high melatonin levels, the opposite of what is expected in healthy individuals [44]. Melatonin can bind to several receptors including MT1 and MT2, which are located in lymphocytes, platelets, prostatic cells, renal tubules, and cardiac myocytes and can also bind to nuclear receptors [45]. Binding to nuclear receptors allows melatonin to alter the transcription of genes and inhibit cell proliferation [45]. Pre-treatments with melatonin in estrogen receptor-alpha (ER alpha positive) MCF-7 human breast cancer cells reduced ER alpha transactivation and binding activity and decreased the elevation of cAMP levels [46]. Decreased cAMP levels suppress the uptake and metabolism of linoleic acid (LA) and results in a reduction of the activation of the epidermal growth factor pathway and thus tumor growth inhibition [47]. In two studies conducted by Lissoni et al., in 1993 [48], melatonin was administered to breast cancer patients to observe the effects on tumor regression and other side effects. Women with metastatic breast cancer who had not responded to tamoxifen (TMX) alone were given TMX at noon and melatonin in the evening. Final reports showed a reduction of lesions and a decrease in insulin-like growth factor (IGF-1) in patients. [45] A second trial was conducted to evaluate the effects of high-dose melatonin in breast cancer patients receiving chemotherapy, which due to its high toxicity limited the frequency of treatment. After four cycles, the melatonin administered was observed to normalize platelet levels in a majority of the test subjects and cause tumor regression in 5 out of 12 patients with little toxicity reported [49].

\section{Melatonin in prostate and colorectal cancer}

Melatonin has also been shown to improve survival rates in patients with prostate and colorectal cancer. Studies have shown that men with primary localized malignant prostate tumors have extremely low levels of nocturnal melatonin that decrease with an increase in tumor growth [50]. Patients with unoperated colorectal carcinoma were found to have a significantly lower nocturnal plasma melatonin level compared to controls [51]. However, a second study conducted by Kvetnaia et al. in 2001 [52] found a higher nocturnal urinary metabolite 6-sulfatoxymelatonin (aMT6s) excretion in operated, untreated males. In one study of 54 patients with metastatic lung and colorectal tumors, the melatonin regimen resulted in stabilization of cancer and improved quality of life for roughly $40 \%$ of the recipients $[42,53]$. As specified previously, the effects of melatonin supplementations can change according to the time of administration. Melatonin injections given in the morning have been found to stimulate cancer growth while injections in the evening contribute to tumor regression. Afternoon injections have no apparent effect [42].

\section{Melatonin in organ specific cancer}

Melatonin has also been shown to affect tumor growth in the thyroid, bronchi, and stomach among other forms of cancer. In female patients with thyroid cancer, the levels of aMT6s were extremely low and did not differ from patients with benign thyroid diseases. This indicates that thyroid growth negatively affects the proper regulation of the pineal gland [53]. In contrast to this finding, Hunt et al. 2001, [54] found significantly elevated levels of aMT6s in thyroid cancer patients [50]. Similarly, several found depressed nocturnal aMT6s-excretion in early stage male bronchial cancer patients while others reported high morning and nighttime melatonin levels of late-stage patients $[55$, 
56]. Furthermore, male patients with primary, unoperated stomach cancer displayed a depletion of the nocturnal urinary excretion of aMT6s. Results from other melatonin-cancer studies achieved similar results: some studies reported reduced nocturnal aMT6s levels while other studies reported high levels. Results from various studies show that melatonin has an apparent effect on cancer development, but the discrepancies among the studies prompt further research into the relationship between melatonin and cancer.

\section{Relationship between melatonin and cancer risk}

With industrialized society drifting more towards a life situated in the presence of 24-hour light, the occurrence of sleep disorders and abnormal sleep patterns continues to increase. As a result, circadian rhythms regulating cellular and physiological processes, such as the secretion of melatonin, have taken a significant toll on the ability to synchronize to the environment. Extended periods of exposure to light further inhibit the production and secretion of melatonin from the pineal gland through proteasomal proteolysis. Decreased endogenous secretion of melatonin has been shown through various clinical and mouse studies and has been linked to a higher risk of certain types of cancer. Recent epidemiological studies have shown that women working night shifts are at a greater risk of breast, endometrial, and colorectal cancer while male night shift workers are at a significantly increased risk of developing prostate cancer presumably due to their increased exposure to light at night [47]. Individuals with various tumor types exhibited depressed nocturnal melatonin concentrations or nocturnal excretion of the main metabolite, 6-sulfatoxymelatonin [57]. There has been a particularly close link between melatonin levels and the risk of breast cancer. Data collected from the women involved in epidemiological studies included urinary melatonin levels (measuring specifically levels of 6- sulfatoxymelatonin), sleep duration, and shift work [58]. The results from the many studies conducted throughout the world, however, displayed contradicting information. Breast cancer risk was found to be elevated with decreased urinary melatonin levels in the Nurses' Health Study II, but results from the UK Guernsey Cohort study showed very little significant association between levels of urinary melatonin and the risk of breast cancer $[59,60]$.

\section{Melatonin and the prevention of cancer growth}

Cancer patients in controlled trials conducted by Wang et al. further showed that melatonin significantly improved complete and partial remission, was associated with a 1-year survival rate, and dramatically decreased radiochemotherapy-related side effects including thrombocytopenia, neurotoxicity, and fatigue [61]. Mouse studies have demonstrated the inhibition of the development of breast cancer models by melatonin as well as the growth of tumors in rodents with exposure to long periods of light via a melatonininduced suppression of tumor linoleic acid uptake and its metabolism to the mitogenic signaling molecule 13HODE. [62] Due to melatonin's ability to suppress cAMP formation, it inhibits the uptake of LA and its metabolism to 13-HODE by a MEL receptor-mediated mechanism found in rat hepatomas and human breast cancer xenografts [62]. Endothelin-1 (EDN1) is an angiogenic factor that promotes the growth of tumors in the blood vessels. To combat this factor, melatonin inhibits the synthesis of endothelin-1 to block the growth of cancer cells [45]. There is evidence that the administration of melatonin alone or in combination with interleukin-2 or chemo-radiotherapy in patients with advanced tumors is associated with tumor regression and improved survival rates [63]. Melatonin prevents the proliferation of cancer cells by reducing telomere length and telomerase activity, both of which are responsible for the development of unhealthy cells and high levels of the hormone, suppressing the tumorpromoting gene TP53 [45]. Additional data from clinical studies further establishes that the use of melatonin has minor side effects, and thus it is a hormone growing in popularity for the development of cancer treatments.

\section{Adverse effects of melatonin as an anti-cancer treatment:}

Clinical trials using melatonin as an anticancer treatment and studies conducted on the role of melatonin in the prevention of cancer development show the surging potential of melatonin as a therapeutic medication to combat sleep disorders, cancer, and the side effects of chemotherapy. As seen in both mammals and humans, melatonin has a very low level of toxicity, and even in relatively high doses, it typically does not cause major side effects [42]. Minor side effects, however, include headaches, dizziness, and drowsiness as well as nausea and apathy combined with weight gain $[64,65]$. Melatonin treatment in children, however, can be sustained long-term without severe deviations of normal development, such as sleep quality, puberty, and mental health [65]. Although, melatonin has been successfully used in cancer and sleep disorder treatments, improper timing of use can result in negative outcomes. Melatonin injections in the morning can stimulate tumor growth; doses in the afternoon exhibit no effect, and doses in the evening have retarding effects [42]. Studies have shown that large doses of melatonin increased light-induced damage to retinal photoreceptors (ganglion cells, rods, and cones) [66]. Furthermore, administration of melatonin that elongates the normal nocturnal melatonin pattern has been shown to exacerbate Seasonal Affected Disorders (SADs), bipolarity, and classic depression [42]. Melatonin is an easily accessible drug provided as an over-the-counter supplement. With its various roles in physiology and cellular processes, it also can interact with many other drugs, such as anticoagulants, interleukin-2, and antidepressant medications [66]. Studies show that melatonin can increase the risk of bleeding from anticoagulant medications such as 
warfarin, increase tumor regression and survival rates of cancer in conjunction with IL-2, and reduce antidepressant effects of drugs such as desipramine $[67$, 68].

\section{CONCLUSION}

The pineal hormone and drug supplement, melatonin, plays a major role in maintaining circadian rhythms throughout the body. Not only is it involved in the normalizing of biological rhythms, but it is also observed to function in the immune system, in the reproductive system, as an antioxidant, and as an anticancer treatment. Through a series of apoptosis pathways and interactions with the immune system, chemotherapy, and anti-cancer drugs, melatonin can help increase tumor regression in cancer patients. Melatonin has been shown to induce many positive effects to protect the body and cells from damage, but improper administration can culminate in hazardous effects, including the stimulation of tumor growth. The studies conducted until now have shown great potential for the clinical use of melatonin to target certain diseases; however, more research must be completed to establish the precise role of melatonin in the body and its range of effects on physiology and behavior.

\section{REFERNCES}

1. Lerner, A.B., Case, J.D., Takahashi, Y. (1958). Isolation of melatonin, a pineal factor that lightens melanocytes. J Am Chem Soc, 80: 2587.

2. Axelrod, J., Wurtman, R.J. (1968). Photic and neural control of indoleamine metabolism in the rat pineal gland. Adv Pharmacol, 6: 157-66.

3. Tricoire, H., Moller, M., Chemineau, P. (2003). Origin of cerebrospinal fluid melatonin and possible function in the integration of photoperiod. Reprod (Suppl), 61: 311-21.

4. Cardinali, D.P., Pevet, P. (1998). Basic aspects of melatonin action. Sleep Med Rev, 2: 175-90.

5. Claustrat, B., Brun, J., Chazot, G. (2005). The basic physiology and pathophysiology of melatonin. Sleep Med Rev, 9: 11-4.

6. Skene, D.J., Papagiannidou, E., Hashemi, E. (2001). Contribution of CYP1A2 in the hepatic metabolism of melatonin: studies with isolated microsomal preparations and liver slices. J Pineal Res, 31: 333-42.

7. Hardeland, R., Reiter, R.J., Poeggeler, B. (1993). The significance of the metabolism of the neurohormone melatonin: antioxidative protection and formation of bioactive substances. Neurosci Biobehav Rev, 17: 347-57.

8. Tan, D. X., Manchester, L. C., Terron, M. P., Flores, L. J., \& Reiter, R. J. (2007). One molecule, many derivatives: a never- ending interaction of melatonin with reactive oxygen and nitrogen species?. Journal of pineal research, 42(1), 28-42.

9. Tan, D.X., Manchester, L.C., Reiter, R.J. (2000). Significance of melatonin in antioxidative defense system: reactions and products. Biol Signals Recept, 9:137-9.

10. Klein, D.C., Moore, R.Y. (1979). Pineal Nacetyltransferase and hydroxyindole-Omethyltransferase: control by the retinohypothalamic tract and the suprachiasmatic nucleus. Brain Res, 174: 245-2.

11. Berson, D.M., Dunn, F.A., Takao, M. (2002). Phototransduction by retinal ganglion cells that set the circadian clock. Science, 295: 1070-3.

12. Buijs, R.M., Hermes, M.H., Kalsbeek, A. (1998). The suprachiasmatic nucleus paraventricular nucleus interactions: a bridge to the neuroendocrine and autonomic nervous system. Prog Brain Res, 119: 365-2.

13. Klein, D.C., Weller, J.L., Moore, R.Y. (1971). Melatonin metabolism: neural regulation of pineal serotonin: acetyl coenzyme A N-acetyltransferase activity. Proc NatL Acad Sci, USA, 68: 3107-10.

14. Vacas, M.I., Lowenstein P, Cardinali DP. Dihydroergocryptine binding sites in bovine and rat pineal glands. J Auton Nerv Syst 1980; 2: 305-3.

15. Ho, A. K., \& Klein, D. C. (1987). Activation of alpha 1-adrenoceptors, protein kinase $\mathrm{C}$, or treatment with intracellular free $\mathrm{Ca} 2+$ elevating agents increases pineal phospholipase A2 activity. Evidence that protein kinase $\mathrm{C}$ may participate in Ca2+-dependent alpha 1-adrenergic stimulation of pineal phospholipase A2 activity. Journal of Biological Chemistry, 262(24), 11764-11770.

16. Krause, D. N., \& Dubocovich, M. L. (1990). Regulatory sites in the melatonin system of mammals. Trends in neurosciences, 13(11), 464470.

17. Maronde, E., \& Stehle, J. H. (2007). The mammalian pineal gland: known facts, unknown facets. Trends in Endocrinology \& Metabolism, 18(4), 142-149.

18. SCHOMERUS, C., \& KORF, H. W. (2005). Mechanisms regulating melatonin synthesis in the mammalian pineal organ. Annals of the New York Academy of Sciences, 1057(1), 372-383.

19. Ganguly, S., Weller, J. L., Ho, A., Chemineau, P., Malpaux, B., \& Klein, D. C. (2005). Melatonin synthesis: 14-3-3-dependent activation and inhibition of arylalkylamine $\mathrm{N}$-acetyltransferase mediated by phosphoserine-205. Proceedings of the National Academy of Sciences, 102(4), 12221227.

20. Gastel, J. A., Roseboom, P. H., Rinaldi, P. A., Weller, J. L., \& Klein, D. C. (1998). Melatonin production: proteasomal proteolysis in serotonin Nacetyltransferase regulation. Science, 279(5355), 1358-1360.

21. Cardinali, D. P., \& Rosner, J. M. (1971). Metabolism of serotonin by the rat retina in vitro. Journal of neurochemistry, 18(9), 17691770.

22. NT, R. (1976). Kvetnoy IM. Melatonin and enterochromaffin cells. Acta Histochem, 55, 19-24. 
23. Slominski, A., Fischer, T. W., Zmijewski, M. A., Wortsman, J., Semak, I., Zbytek, B., ... \& Tobin, D. J. (2005). On the role of melatonin in skin physiology and pathology. Endocrine, 27(2), 137147.

24. Carrillo- Vico, A., Calvo, J. R., Abreu, P., Lardone, P. J., García- Mauriño, S., Reiter, R. J., \& Guerrero, J. M. (2004). Evidence of melatonin synthesis by human lymphocytes and its physiological significance: possible role as intracrine, autocrine, and/or paracrine substance. The FASEB Journal, 18(3), 537-539.

25. Conti, A., Conconi, S., Hertens, E., SkwarloSonta, K., Markowska, M., \& Maestroni, G. J. (2000). Evidence for melatonin synthesis in mouse and human bone marrow cells. Journal of pineal research, 28(4), 193-202.

26. Cardinali, D. P., Furio, A. M., Reyes, M. P., \& Brusco, L. I. (2006). The use of chronobiotics in the resynchronization of the sleep-wake cycle. Cancer Causes \& Control, 17(4), 601-609.

27. Pandi-Perumal, S. R., Zisapel, N., Srinivasan, V., \& Cardinali, D. P. (2005). Melatonin and sleep in aging gerontology, 40(12), 911-925.

28. Reiter, R. J. (1980). The pineal and its hormones in the control of reproduction in mammals. Endocrine reviews, 1(2), 109-131.

29. Lincoln, G. A. (2006). Melatonin entrainment of circannual rhythms. Chronobiology international, 23(1-2), 301-306.

30. Gupta, B. B. P., Spessert, R., \& Vollrath, L. (2005). Molecular components and mechanism of adrenergic signal transduction in mammalian pineal gland: regulation of melatonin synthesis.

31. Zisapel, N. (2001). Melatonin-dopamine interactions: from basic neurochemistry to a clinical setting. Cellular and molecular neurobiology, 21(6), 605-616.

32. Karasek, M. (2004). Melatonin, human aging, and age-related diseases. Experimental gerontology, 39(11-12), 1723-1729.

33. Salti, R., Galluzzi, F., Bindi, G., Perfetto, F., Tarquini, R., Halberg, F., \& Cornelissen, G. (2000). Nocturnal melatonin patterns in children. The Journal of Clinical Endocrinology \& Metabolism, 85(6), 2137-2144.

34. Scheer, F. A., Van Montfrans, G. A., van Someren, E. J., Mairuhu, G., \& Buijs, R. M. (2004). Daily nighttime melatonin reduces blood pressure in male patients with essential hypertension. Hypertension, 43(2), 192-197.

35. Grossman, E., Laudon, M., Yalcin, R., Zengil, H., Peleg, E., Sharabi, Y., ... \& Zisapel, N. (2006). Melatonin reduces night blood pressure in patients with nocturnal hypertension. The American journal of medicine, 119(10), 898-902.

36. Tan, D. X., Manchester, L. C., Di Mascio, P., Martinez, G. R., Prado, F. M., \& Reiter, R. J. (2007). Novel rhythms of N1- acetyl- N2- formyl- 5- methoxykynuramine and its precursor melatonin in water hyacinth: importance for phytoremediation. The FASEB Journal, 21(8), 1724-1729.

37. Gorfine, T., \& Zisapel, N. (2007). Melatonin and the human hippocampus, a time dependant interplay. Journal of pineal research, 43(1), 80-86.

38. Girotti, L., Lago, M., Ianovsky, O., Elizari, M. V., Dini, A., Lloret, S. P., ... \& Cardinali, D. P. (2003). Low urinary 6-sulfatoxymelatonin levels in patients with severe congestive heart failure. Endocrine, 22(3), 245-248.

39. Pandi-Perumal, S. R., Srinivasan, V., Poeggeler, B., Hardeland, R., \& Cardinali, D. P. (2007). Drug insight: the use of melatonergic agonists for the treatment of insomnia - focus on ramelteon. Nature Clinical Practice Neurology, 3(4), 221-228.

40. Leger, D., Laudon, M., \& Zisapel, N. (2004). Nocturnal 6-sulfatoxymelatonin excretion in insomnia and its relation to the response to melatonin replacement therapy. The American journal of medicine, 116(2), 91-95.

41. Bartsch, C., \& Bartsch, H. (2006). The anti-tumor activity of pineal melatonin and cancer enhancing life styles in industrialized societies. Cancer Causes \& Control, 17(4), 559-571.

42. Malhotra, S., Sawhney, G., \& Pandhi, P. (2004). The therapeutic potential of melatonin: a review of the science. Medscape General Medicine, 6(2).

43. Anisimov, V. N., Alimova, I. N., Baturin, D. A., Popovich, I. G., Zabezhinski, M. A., Manton, K. G., ... \& Yashin, A. I. (2003). The effect of melatonin treatment regimen on mammary adenocarcinoma development in HER- 2/neu transgenic mice. International journal of cancer, 103(3), 300-305.

44. Bartsch, C., Bartsch, H., Jain, A. K., Laumas, K. R., \& Wetterberg, L. (1981). Urinary melatonin levels in human breast cancer patients. Journal of neural transmission, 52(4), 281-294.

45. Agrawal, A., Darbari, S., Rai, T. P., \& Kulkarni, G. T. (2016). Role of Melatonin in the Pathophysiology of Cancer. J Chron DD, 7, 1-6.

46. Kiefer, T., Ram, P. T., Yuan, L., \& Hill, S. M. (2002). Melatonin inhibits estrogen receptor transactivation and cAMP levels in breast cancer cells. Breast cancer research and treatment, 71(1), 37-45.

47. Blask, D. E. (2009). Melatonin, sleep disturbance and cancer risk. Sleep medicine reviews, 13(4), 257-264.

48. Lissoni, P., Barni, S., Tancini, G., Ardizzoia, A., Rovelli, F., Cazzaniga, M., \& Characiejus, D. (1993). Immunotherapy with subcutaneous lowdose interleukin-2 and the pineal indole melatonin as a new effective therapy in advanced cancers of the digestive tract. British journal of cancer, 67(6), 1404-1407.

49. Kaczor, T. (2010). An overview of melatonin and breast cancer. Nat Med Journal, 2:2 
50. Bartsch, C., Bartsch, H., Fluechter, S. H., \& Lippert, T. H. (1993, January). Depleted pineal melatonin production in patients with primary breast and prostate cancer is connected with circadian disturbances of central hormones: possible role of melatonin for maintenance and synchronization of circadian rhythmicity. In excerpta medica international congress series (Vol. 1017, No. 1, pp. 311-311). Elsevier.

51. Khoory, R., \& Stemme, D. (1988). Plasma melatonin levels in patients suffering from colorectal carcinoma. Journal of pineal research, 5(3), 251-258.

52. Kvetnaia, T.V., Kvetnoy, I.M., Bartsch, H. (2001). Melatonin in cancer with extra-reproductive location. In: Bartsch C, Bartsch H, Blask DE, eds. The Pineal Gland and Cancer: Neuroimmunoendocrine Mechanisms in Malignancy. Berlin: Springer, 177-196.

53. Dogliotti, L., Berruti, A., Buniva, T. (1990). Melatonin and human cancer. J Steroid Biochem Mol Biol, 37: 983-7.

54. Hunt, A. E., Al-Ghoul, W. M., Gillette, M. U., \& Dubocovich, M. L. (2001). Activation of MT2 melatonin receptors in rat suprachiasmatic nucleus phase advances the circadian clock. American Journal of Physiology-Cell Physiology,280(1), C110-C118.

55. Viviani, S., Bidoli, P., Spinazze, S., Rovelli, F., \& Lissoni, P. (1992). Normalization of the light/dark rhythm of melatonin after prolonged subcutaneous administration of interleukin- 2 in advanced small cell lung cancer patients. Journal of pineal research, 12(3), 114-117.

56. Dogliotti, L., Berruti, A., Buniva, T., Torta, M., Bottini, A., Tampellini, M., ... \& Angeli, A. (1990). Melatonin and human cancer. The Journal of steroid biochemistry and molecular biology, 37(6), 983-987.

57. Sánchez-Hidalgo, M., M Guerrero, J., Villegas, I., Packham, G., \& A de la Lastra, C. (2012). Melatonin, a natural programmed cell death inducer in cancer. Current medicinal chemistry, 19(22), 3805-3821.

58. Rondanelli, M., Faliva, M. A., Perna, S., \& Antoniello, N. (2013). Update on the role of melatonin in the prevention of cancer tumorigenesis and in the management of cancer correlates, such as sleep-wake and mood disturbances: review and remarks. Aging clinical and experimental research, 25(5), 499-510.

59. Schernhammer, E. S., \& Hankinson, S. E. (2005). Urinary melatonin levels and breast cancer risk. Journal of the National Cancer Institute, 97(14), 1084-1087.

60. Travis, R. C., Allen, D. S., Fentiman, I. S., \& Key, T. J. (2004). Melatonin and breast cancer: a prospective study. Journal of the National Cancer Institute, 96(6), 475-482.

61. Wang, Y. M., Jin, B. Z., Ai, F., Duan, C. H., Lu, Y. Z., Dong, T. F., \& Fu, Q. L. (2012). The efficacy and safety of melatonin in concurrent chemotherapy or radiotherapy for solid tumors: a meta-analysis of randomized controlled trials. Cancer chemotherapy and pharmacology, 69(5), 1213-1220.

62. Blask, D. E., Dauchy, R. T., \& Sauer, L. A. (2005). Putting cancer to sleep at night. Endocrine, 27(2), 179-188.

63. Cutando, A.1., López-Valverde, A., AriasSantiago, S. (2012). Role of Melatonin in Can Treat Antican Res, 32: 2747-53.

64. [Internet] Bauer, B. A. (2015). Melatonin side effects: What are the risks? https://forum.psychlinks.ca/showthread.php?32015 -Melatonin-side-effects-What-are-the-risks.

65. Van Geijlswijk, I. M., Mol, R. H., Egberts, T. C., \& Smits, M. G. (2011). Evaluation of sleep, puberty and mental health in children with long-term melatonin treatment for chronic idiopathic childhood sleep onset insomnia. Psychopharmacology, 216(1), 111-120.

66. Wiechmann, A. F., \& O'Steen, W. K. (1992). Melatonin increases photoreceptor susceptibility to light-induced damage. Investigative ophthalmology \& visual science, 33(6), 1894-1902.

67. [Internet] Penn State Hershey. Possible Interactions with: Melatonin. http://pennstatehershey.adam.com/content.aspx?pr oductId=107\&pid=33\&g id=000970

68. [Internet] University of Maryland Medical Center. Possible Interactions with: Melatonin. https://www.umm.edu/health/medical/altmed/suppl ement-interaction/possible-interactions-withmelatonin. 